\title{
Téoros
}

Revue de recherche en tourisme

\section{Le tourisme sénégalais à la recherche d'une nouvelle identité}

\section{Mamadou Diombéra}

Volume 31, numéro 2, 2012

URI : https://id.erudit.org/iderudit/1020768ar

DOI : https://doi.org/10.7202/1020768ar

Aller au sommaire du numéro

\section{Éditeur(s)}

Université du Québec à Montréal

\section{ISSN}

0712-8657 (imprimé)

1923-2705 (numérique)

Découvrir la revue

Citer cet article

Diombéra, M. (2012). Le tourisme sénégalais à la recherche d'une nouvelle identité. Téoros, 31(2), 21-30. https://doi.org/10.7202/1020768ar

\section{Résumé de l'article}

Le secteur du tourisme au Sénégal s’est imposé comme le véritable moteur de l'économie, au second rang après la pêche. Développé aussitôt après les indépendances, le tourisme international et essentiellement balnéaire, appréhendé comme un facteur de développement, n’a cessé de croître. Les arrivées massives de touristes, notamment de type tout inclus, ont quasiment quadruplé de 1960 à 2000. Les transactions touristiques ont atteint, durant la même période, près de $10 \%$ de la valeur globale du commerce extérieur du pays. Malheureusement, ces dernières années, la destination est fortement concurrencée et son image, stigmatisée par la mauvaise réputation de la station balnéaire de Saly Portudal. Face à une telle situation qui devient de plus en plus exécrable, les autorités sénégalaises tentent éperdument, depuis bientôt une décennie, de trouver des éléments de réponse à la lancinante problématique du tourisme de masse par la diversification de l'offre à travers la mise en place de nouvelles formes alternatives de tourisme. 


\title{
Le tourisme sénégalais à la recherche d'une nouvelle identité
}

\author{
Mamadou DIOMBÉRA, Ph.D. \\ Enseignant-chercheur en Tourisme \\ Unité de Formation et de Recherche en Sciences Économiques et Sociales \\ Université Assane Seck de Ziguinchor, Sénégal \\ mamadoudiombera70@yahoo.fr
}

\begin{abstract}
RÉSUMÉ: Le secteur du tourisme au Sénégal s'est imposé comme le véritable moteur de l'économie, au second rang après la pêche. Développé aussitôt après les indépendances, le tourisme international et essentiellement balnéaire, appréhendé comme un facteur de développement, n'a cessé de croître. Les arrivées massives de touristes, notamment de type tout inclus, ont quasiment quadruplé de 1960 à 2000. Les transactions touristiques ont atteint, durant la même période, près de $10 \%$ de la valeur globale du commerce extérieur du pays. Malheureusement, ces dernières années, la destination est fortement concurrencée et son image, stigmatisée par la mauvaise réputation de la station balnéaire de Saly Portudal. Face à une telle situation qui devient de plus en plus exécrable, les autorités sénégalaises tentent éperdument, depuis bientôt une décennie, de trouver des éléments de réponse à la lancinante problématique du tourisme de masse par la diversification de l'offre à travers la mise en place de nouvelles formes alternatives de tourisme.

Cet article propose une analyse de l'évolution de l'aménagement touristique du littoral et de ses conséquences. II propose ensuite des mesures alternatives pour contrer la baisse vertigineuse des flux touristiques balnéaires.

L'étude repose sur une recherche documentaire et de terrain. La recherche documentaire a permis de recueillir des données statistiques officielles sur la demande touristique en termes de volumes et de valeurs, et celle des enquêtes qualitatives, d'appréhender les images et perceptions des acteurs directement ou indirectement impliqués dans la pratique touristique et de proposer des éléments d'une stratégie globale orientée vers l'écotourisme.
\end{abstract}

Mots-clés: Tout inclus, aménagement, écotourisme, littoral, Sénégal.

Plusieurs pays ont développé, après la Seconde Guerre mondiale, une industrie touristique centrée et dominée par le tourisme balnéaire. Depuis l'éveil environnemental, ce tourisme stagne, accusant même une perte de croissance dans certaines destinations. C’est le cas du Sénégal.

Le tourisme sénégalais repose sur de nombreux atouts. Il résulte de la conjugaison d'une offre très riche et variée et d'une demande réelle mais peu diversifiée. Parmi les divers attraits naturels, géographiques, climatiques et humains, on peut notamment citer un ensoleillement constant pendant toute l'année, une multitude de parcs nationaux et de réserves, une culture riche et variée, grâce à une diversité ethnique et linguistique, et la Téranga symbolisant l'hospitalité légendaire, érigée en valeur sociale nationale. Ce concept est également devenu l'un des éléments clés des slogans publicitaires touristiques : «le Sénégal, Porte ouverte de l'Afrique» y est souvent désigné comme le pays de la Téranga (MTTA, 2007 : 1).
Certes, le développement du tourisme sur le littoral a obéi à une logique de promotion de nouvelles destinations balnéaires «tropicales» pour répondre aux enjeux du tourisme de masse des années 1970. Toutefois, comme le décrit Alain Corbin (1988 : 396), l'espace littoral va faire l'objet de transformations profondes pour passer d' "un état répulsif à un état attractif ». C'est ainsi que plusieurs projets touristiques portés par l'État sont orientés vers cet espace littoral, notamment la Petite-Côte et la Basse-Casamance. Le secteur littoral est désormais conçu comme un réservoir de richesses capable de répondre à une mise en tourisme du territoire. Dans cette perspective, le pays est divisé en zones de développement touristique qui accordent la priorité à quatre pôles littoraux, à savoir Dakar, Saint-Louis, Thiès et la Basse-Casamance (Diombéra, $2010: 2$ ).

En effet, en raison de sa capacité d'attraction des investissements privés et des enjeux financiers majeurs qu'elle 


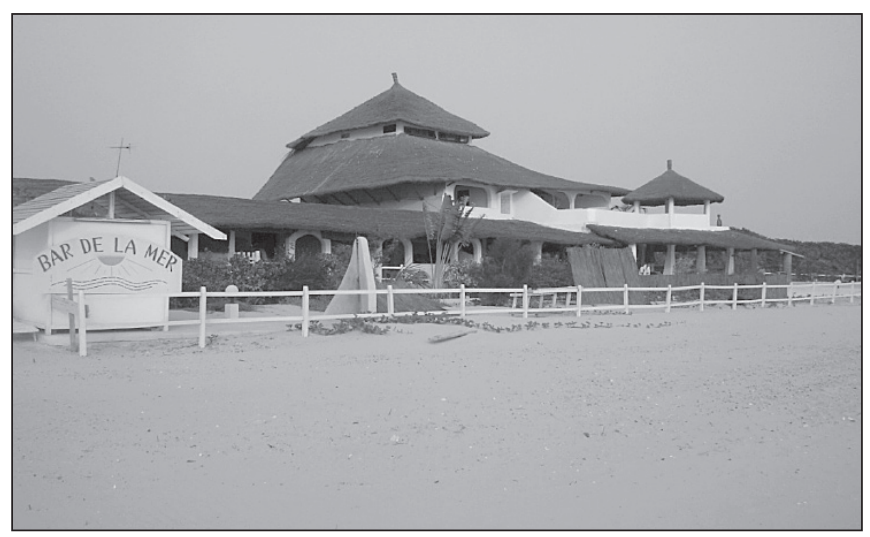

ILLUSTRATION 1 : La résidence «les Alizée » au Cap Skirring

(photo : Mamadou Diombéra).

représente, l'activité touristique va s'inscrire au cœur de la problématique de développement économique et social du Sénégal. Cette volonté du gouvernement sénégalais a été réaffirmée dès 1959 dans le premier plan stratégique de développement économique et social du pays, où elle a été choisie comme levier devant jouer pleinement son rôle de soutien aux finances publiques. Cependant, son développement s'est fortement orienté vers l'aménagement du littoral qui n'offre qu'un produit exclusivement balnéaire qui, après des années de succès éclatant, est entré dans une ère de déclin progressif. Le maintien de l'économie touristique nécessite la mise en place d'une autre forme de tourisme pour relancer l'activité dans cette zone et la rééquilibrer à l'échelle du pays à travers la mise en ouvre d'une nouvelle politique d'aménagement de l'espace et le développement du tourisme en milieu naturel. Ceci se justifie par le fait que le Sénégal doit étendre son activité touristique sur l'ensemble de son territoire et non la confiner uniquement sur le seul littoral. L'autre raison qui pourrait être avancée est que le pays dispose de potentialités naturelles aussi diverses que les bas-fonds dans la zone des Niayes (dépressions interdunaires situées le long de la Grande-Côte Nord, où le maraîchage occupe une bonne place), les mangroves des îles du Saloum et de la Casamance, les étendues de forêts au Sénégal oriental et en Casamance et enfin la présence d'un plan d'eau pérenne le long de la moyenne vallée du fleuve Sénégal qui s'étend de Richard-Toll jusqu’à Bakel.

Devant le tourisme littoral qui connait depuis une quinzaine d'années un recul important au Sénégal et qui soulève de nombreuses questions sur la nécessité de rééquilibrer l'activité, cet article met l'accent sur le besoin de l'industrie du tourisme de se renouveler, à partir de produits alternatifs durables comme l'écotourisme.

Cette problématique s'inscrit dans le champ de recherche de l'aménagement touristique, peu exploré au Sénégal. Elle permet d'appréhender la manière dont le tourisme littoral a participé à la fragilisation de l'écosystème et de la biodiversité qui impose de nouvelles stratégies touristiques autour de l'écotourisme pour redynamiser le secteur.

Pour mener cette étude, nous avons eu recours à deux méthodes de recherche complémentaires. Dans un premier temps, une recherche documentaire a consisté en la consultation d'ouvrages, de documents et de rapports administratifs sur la problématique de développement du tourisme littoral. Cette méthode a permis de recueillir des statistiques fiables pour une analyse quantitative de la demande, de la répartition et de l'évolution du tourisme au Sénégal. Dans un second temps, nous avons entrepris une démarche dite qualitative à travers une quinzaine d'entretiens individuels menés auprès de différents professionnels, des touristes résidents et non résidents des sites touristiques de Saly Portudal et du Cap Skirring. Cette démarche nous a permis de clarifier les différentes typologies d'aménagement, d'approfondir le regard des touristes sur le territoire et d'identifier leurs attentes. Ces informations collectées et analysées selon l'approche systémique ont contribué à définir des relations entre politique d'aménagement et développement touristique, les tendances actuelles et futures pour le développement du tourisme, les possibilités de faire collaborer les sphères politique et sociale, les possibilités de mettre en application les principes du développement durable à travers l'écotourisme.

\section{Un tourisme essentiellement littoral : de l'aménagement de l'État à l'aménagement spontané}

Le Sénégal dispose de $718 \mathrm{~km}$ de plage de sable fin, s'étendant de Saint-Louis à la Casamance. Il se positionne comme la première destination balnéaire en Afrique francophone. Le produit balnéaire se localise surtout dans la Petite-Côte (région de Thiès) avec ses plages aux multiples facettes, comme la station de Saly. Il est aussi présent dans la région de Dakar, qui est une presqu'île, la station de Cap Skirring dans la région de Casamance, sans oublier l'hydrobase de Saint-Louis.

À ses débuts dans les années 1960, le tourisme international de masse a été encouragé par un aménagement touristique littoral dirigé par l'État. Les premières opérations d'aménagement et de planification touristiques devaient apporter des retombées fructueuses dans les régions pauvres. C'est ainsi que des mesures incitatives ont été prises pour soutenir le développement de l'activité par la création d'organismes spécialisés comme la Société financière sénégalaise pour le développement de l'industrie et du tourisme (SOFISEDIT), la Société d'aménagement touristique des côtes et zones touristiques du Sénégal (SAPCO) et l'Agence nationale de la promotion touristique (ANPT).

Le premier organisme a été conçu pour financer le secteur touristique; le second, afin d'aménager la frange littorale pour abriter des unités hôtelières internationales et de faire la promotion des investissements. Le troisième, enfin, a été créé avec la mission de mettre en œuvre la politique du gouvernement dans les domaines de la promotion touristique, de susciter une synergie entre les différents partenaires de l'État dans le développement du secteur, de déterminer et de réaliser des programmes d'actions spécifiques pour la promotion touristique.

Si l'activité balnéaire est ainsi un fait ancien sur le littoral sénégalais, elle est longtemps restée secondaire et limitée à la Petite-Côte. Ce n'est qu'à partir des années 1960 que l'irruption brutale du tourisme de masse va bouleverser l'économie littorale sénégalaise et affecter les activités traditionnelles. 
La mise en tourisme du littoral a connu un développement spectaculaire. C'est dans le contexte de sécheresse des années 1970 qui a affecté la production arachidière en faisant chuter les rentrées de devises du pays que le gouvernement avait pris l'option stratégique de développer l'activité touristique sur le littoral.

Au début des années 1990, l'introduction des résidences para-hôtelières, pour répondre à un souci de diversification de l'offre d'hébergement, a provoqué une recrudescence d'aménagements ponctuels dans un grand nombre de sites littoraux recherchés comme Saly Niakh Niakhal, Somone, Nianing et Palmarin sur la Petite-Côte, et Diembéring, Kabrousse et Abéné en Basse-Casamance. Ces aménagements sont représentés par de gigantesques constructions modernes, constituées en circuit fermé, très convoitées par les touristes (voir illustration 1).

Programmées pour soutenir l'activité touristique, les résidences secondaires se révèlent comme un élément conquérant supplémentaire, de grands «dévorateurs» d'espaces, au point d'étayer la thèse selon laquelle le tourisme est devenu une nouvelle forme de colonialisme. Dans tous les cas, ces aménagements constituent une réelle menace, voire une agression pour l'hôtellerie homologuée dans un secteur déjà en difficulté dominée par la dégradation de l'offre et la baisse vertigineuse de la demande.

En vérité, l'aménagement spontané, sous la pression du tourisme de masse n'épargne actuellement ni l'environnement terrestre ni l'environnement marin. Il touche plusieurs secteurs, plus particulièrement les forêts et les exploitations agricoles dont les superficies se rétrécissent de plus en plus devant la poussée immobilière se caractérisant par une organisation spatiale privilégiant la linéarité de l'aménagement, guidée par le rivage.

D'ailleurs, c'est tout le littoral qui est aujourd'hui en proie au phénomène de «résidentialisation». On y dénombre officiellement, sur la seule localité de la Petite-Côte par exemple, près de 57 résidences secondaires avec une capacité de 954 lits (MTTA, 2007 : 4). En réalité, ce sont plusieurs centaines de réceptifs non répertoriés par l'État qu'on retrouve sur cette zone littorale. Sans plan d'ensemble, elles se développent de manière anarchique. Ces occupations de l'espace côtier, plus particulièrement du front de mer, constituent une sérieuse préoccupation du point de vue de la répartition spatiale et de l'occupation des sols. C'est pourquoi le littoral est devenu la véritable zone problème en termes de contrôle foncier. Des résidences secondaires ou particulières isolées s'y créent $e x$ nihilo sans le moindre contrôle ni le moindre respect des lois et règlements en vigueur en matière d'occupation du sol et du rivage, en provoquant le gonflement et le bourgeonnement des villes et des villages périphériques. C'est ce que montre l'illustration 2 sur la répartition des résidences secondaires par région où on note leur forte concentration sur les principales villes littorales du pays avec près de $60 \%$.

La mise en tourisme de masse du littoral crée un nouveau rapport avec la mer. Cet espace littoral est devenu, en quelques décennies, une zone d'attraction touristique majeure. Les pratiques touristiques occidentales qui s'y développent, fortement liées à des formes paysagères, sont en train de

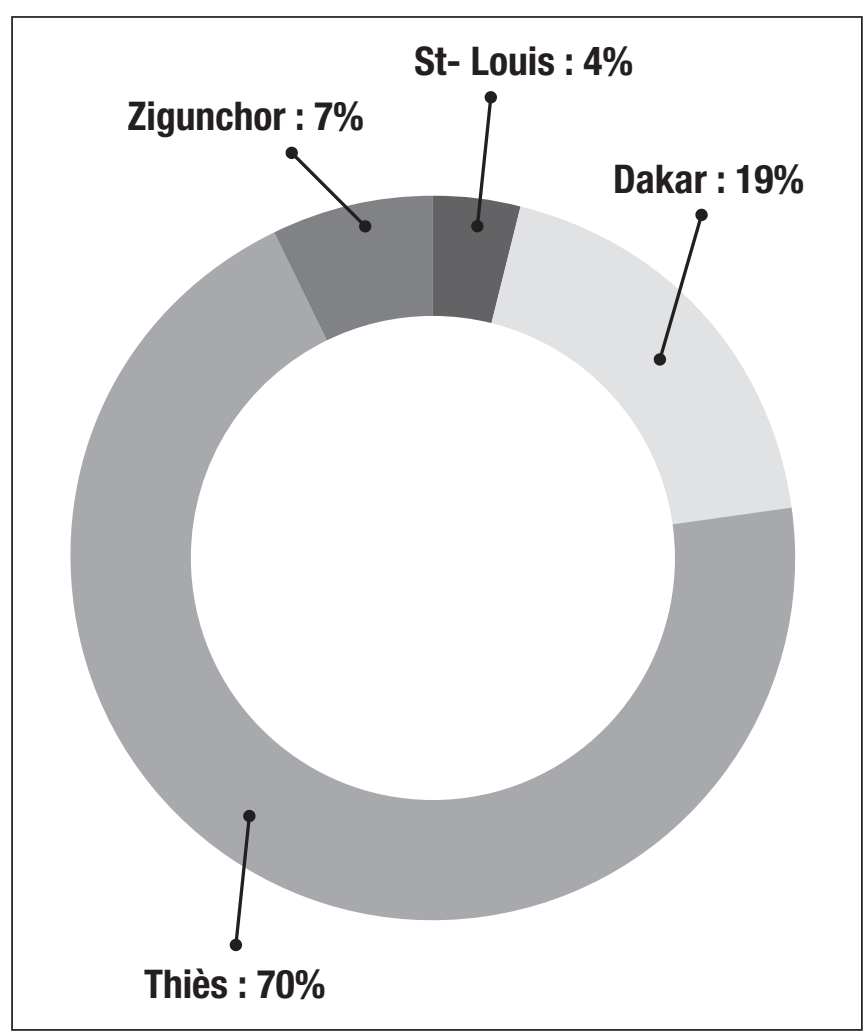

ILLUSTRATION 2 : Répartition des résidences par région (2006)

(source : Ministère du Tourisme et des Transports Aériens (2007)).

décliner, voire de disparaître. Dans ce contexte, peut-on parler de crise touristique ou de crise de l'espace? Dans tous les cas, le tourisme au Sénégal demeure une activité déséquilibrée géographiquement. À titre d'exemple, en 2006, sur les 814640 touristes qui ont visité le pays, $80 \%$ ont été accueillis sur le seul littoral qui abrite la plus importante capacité litière du pays (MTTA, $2007: 2$ ).

La carte touristique du pays (voir illustration 3 ) indique que l'essentiel des intenses concentrations touristiques se situent principalement sur le littoral (Saint-Louis, Dakar, Delta du Saloum et Basse-Casamance). Ces différents pôles touristiques littoraux offrent des produits, notamment le tourisme balnéaire et de plaisance sportive, aux dépens des autres formes de tourisme comme le tourisme rural intégré, le tourisme culturel et l'écotourisme.

En effet, tous les plans de développement touristique du Sénégal sont orientés sur le littoral. Il en est de même de l'étude des trois importants projets d'aménagement touristiques (Pointe Sarène, Mbodienne et Joal Finio) qui a été bouclée par la Sapco depuis bientôt une décennie. La réalisation de ces projets tarde à voir le jour pour plusieurs causes. Entre autres raisons avancées, il y a l'insuffisance des budgets mis en place par l'État, le peu d'intérêt de la part des investisseurs privés à cause de la saturation du milieu et le déficit chronique en ressources humaines qualifiées (Diombéra, $2010: 238$ ).

Dans la pratique, tous les plans d'aménagement et de planification touristiques n'ont pas été respectés comme 


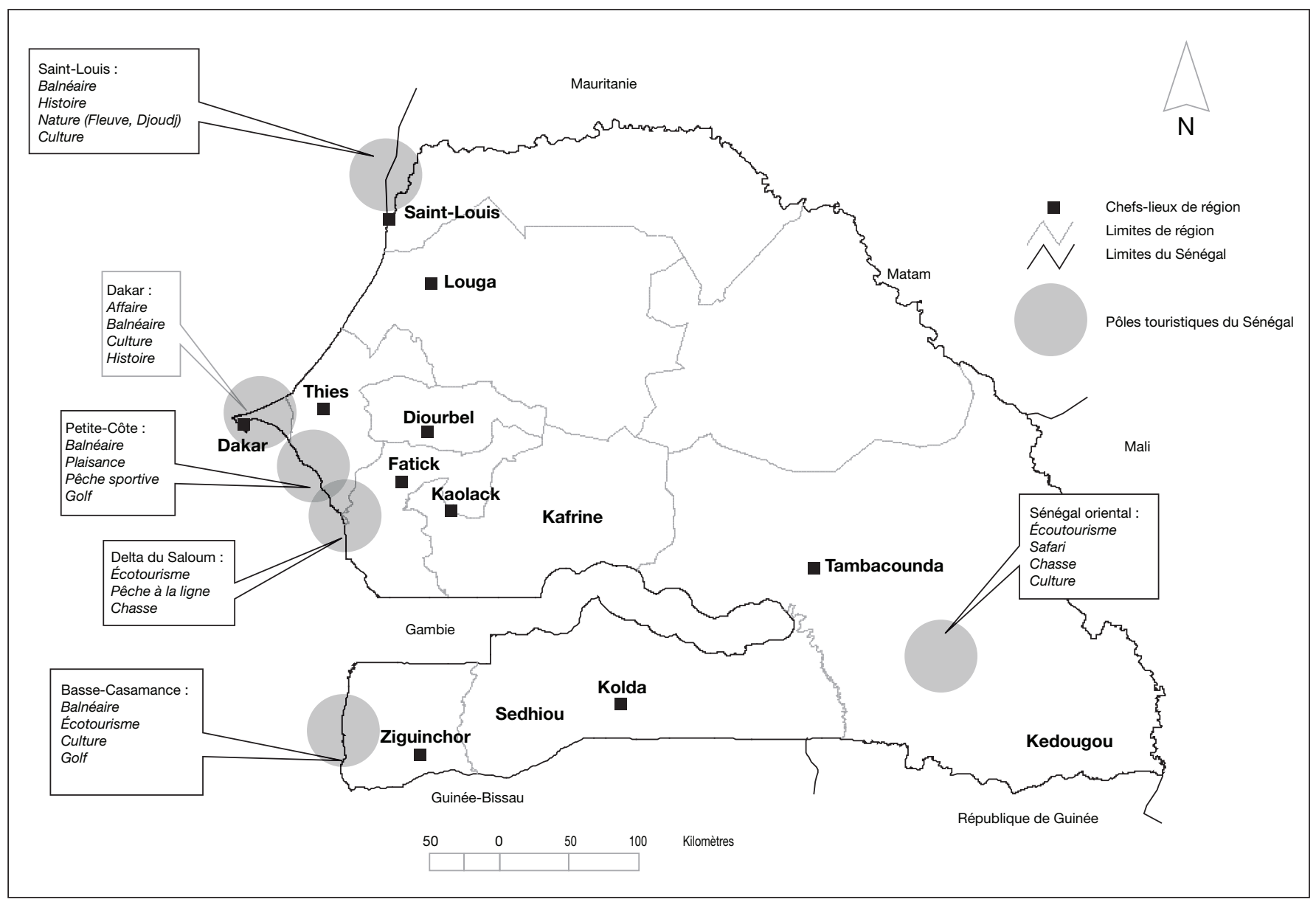

ILLUSTRATION 3 : Les pôles de développement touristiques du Sénégal (source : compilation de l'auteur à partir des données de DTGC (2010)).

initialement prévu. À ce jour, seule la station balnéaire de Saly Portudal a fait l'objet d'un aménagement avec des infrastructures de base (voirie et réseaux divers). L'une des conséquences est l'accélération des déséquilibres à l'échelle du territoire, tant sur le plan socio-économique que spatial.

Les potentialités touristiques des régions littorales sont surexploitées au moment où l'arrière-pays, avec ses immenses richesses touristiques, demeure peu mis en valeur. Les actions d'aménagement, de défrichement et de terrassement des zones touristiques situées au niveau de la plage, du littoral ou de la mangrove sont de nature à favoriser la dégradation insidieuse du milieu en perturbant le fragile équilibre et la vie des espèces faunistiques et floristiques in situ. Ces facteurs anthropiques contribuent, au même titre que les facteurs naturels, à la fragilisation du littoral avec comme conséquence la dégradation de la biodiversité.

L'image du Sénégal étant trop orientée «soleil d'hiver» (Diombéra, $2010: 33$ ), la destination se vend davantage grâce à la promotion de son environnement littoral que par le biais d'attributs «typiques» (Quashie, $2009: 527)$. Dans un environnement très concurrentiel où de nouvelles destinations comme le Cap-Vert attirent de plus en plus de clientèles balnéaires, la tâche de promouvoir ce produit devient alors difficile pour le pays. C'est d'autant plus vrai que pratiquement tous les pays d'Afrique en général et de l'Afrique de l'Ouest en particulier ont pris conscience de l'importance de cette activité dans la croissance de leur commerce extérieur. Dans ces conditions, il est impératif d'être à l'écoute de la clientèle et de l'ensemble des professionnels du secteur et des populations locales pour trouver de nouvelles formes alternatives de tourisme. De fait, il apparaît normal de rassembler les différents atouts et de valoriser la diversité de l'offre. Les différents investissements publics comme privés doivent être plus cohérents. Les efforts financiers doivent être également mieux répartis entre les différents partenaires.

Derrière l'apparente diversification des structures d'accueil se cache une monotonie de l'offre dominée par le balnéaire. Les styles architecturaux se ressemblent. De même, toutes les catégories d'hébergement comme les hôtels haut de gamme, les résidences, les auberges ou les campements offrent la même panoplie d'activités sportives et distractives (piscine, tennis, planche à voile, ski nautique et discothèque). Les plus luxueux vont jusqu'à proposer de la thalassothérapie et surtout du golf, comme en témoigne le nombre impressionnant de brochures touristiques des voyagistes qui commercialisent la destination. Leur argumentaire pour les vacances repose essentiellement 
sur un discours «rêveur» d'un paradis récréatif sur le littoral (Amirou, 1995 : 13) plus que sur la découverte de l'arrièrepays ou d'une région authentique.

\section{Le tourisme littoral : une panacée de développement économique?}

Le tourisme est aujourd'hui à la base de la croissance économique de la Petite-Côte et de la Basse-Casamance. En l'espace d'un quart de siècle, il a su s'imposer comme la deuxième source de revenus du pays après la pêche. Les flux touristiques, essentiellement balnéaires, représentent près de $54 \%$ des arrivées touristiques au Sénégal et assurent l'essentiel des retombées économiques du tourisme (MTTA, 2007 : 2). Selon les estimations du ministère de l'Économie et des Finances (MEF, 2003 : 26), le produit intérieur brut (PIB) du pays a progressé en moyenne de plus de $5 \%$ par an depuis 1995. Cette performance est imputable à l'amélioration du cadre macro-économique et règlementaire, et surtout à la stratégie de développement du secteur privé (MTTA, 2005 : 5).

L'intérêt du tourisme sur l'économie sénégalaise, dans le contexte actuel, est particulièrement apprécié. Il génère une manne financière importante à l'économie par les consommations, les subventions et les investissements extérieurs. La part du tourisme dans le PIB est de l'ordre de 2,5 \% (MEF, 2003 : 26). Ainsi, avec plus de 101,4 milliards de francs CFA en 1999 à environ 234 milliards en 2004 (OMT, 2003 : 45), le secteur du tourisme (balnéaire) demeure le second pourvoyeur de recettes en devises après la pêche. En outre, les recettes fiscales provenant du tourisme littoral représentent près de $75 \%$ des recettes globales générées par le secteur (MTTA, 2007 : 7). En effet, sur les 25000 emplois directs et 75000 emplois indirects créés par l'activité touristique, les trois quarts seraient localisés en milieu littoral (OMT, 2003 : 45). Le tourisme apparaît alors dans cette zone comme un instrument de lutte efficace contre la pauvreté et pour la promotion des femmes qui occupent en grand nombre des positions subalternes dans les hôtels et autres infrastructures touristiques.

En dépit de ces résultats, on ne doit pas néanmoins sousestimer les effets négatifs de la croissance du tourisme dans ce milieu littoral. S’il est indéniable que le tourisme balnéaire peut être créateur de richesses et d'emplois, cet effet positif doit être examiné avec attention.

\section{Des stations littorales agonisantes : un tourisme mal organisé en perte de vitesse}

Il est bien connu que les voyagistes qui commercialisent le Sénégal proposent des voyages à forfait s'appuyant sur leurs hôtels, favorisant la multiplication des grands hôtels haut ou moyen de gamme, les fameuses usines à vacances (Dumas, 1980 : 193).

Les hôtels de bord de mer forment des plates-formes en contrebas qui ne sont accessibles qu'à leurs clients. La construction massive d'unités hôtelières le long du rivage exclut la population autochtone à l'accès à la mer et à la plage qui deviennent des «propriétés privées» (Paskoff, 1998 : 32). Ce sont là autant de facteurs qui nécessitent de reconsidérer les logiques touristiques et les stratégies de développement de ce secteur d'activité au Sénégal. En effet, l'incursion de l'activité touristique sur le littoral sénégalais rappelle trois attitudes complémentaires qui se traduisent en termes d'anarchie dans laquelle elle s'est développée, de son coût économique du fait de sa concentration dans l'espace et l'inflation qui s'en suit, et de son coût social par les atteintes culturelles et paysagères et la privatisation de l'espace public qu'elle entraîne (Dewailly, 2005 : 23).

Le bilan du tourisme de masse au Sénégal est plus que mitigé (Faye, 2005 : 18). Il a des coûts socioculturels non négligeables sur les sociétés d'accueil. Il est considéré comme une source de pédophilie, de proxénétisme, de prostitution, de toutes les tares et déviances (Faye, 2005 :18). Ce modèle touristique longtemps construit sur les trois « $\mathrm{S}$ » (sun, sand, sea) est en passe de faire croire que le Sénégal, plus particulièrement les stations littorales, sont des destinations du tourisme sexuel. En effet, l'écart se creuse entre ce que les touristes sont supposés venir chercher au Sénégal - à savoir des belles plages et une culture accueillante - et ce qu'ils y trouvent, comme en témoigne la perception insistante selon laquelle Saly Portudal serait devenue trop «touristique».

Selon Dème (2002:11), le tourisme balnéaire sénégalais entraîne 12 catégories de coûts socio-économiques. Il s'agit des coûts des biens et services, du foncier, du capital, de la dépendance économique à travers l'importation d'équipements, du social, de l'emploi, du budget et de la fiscalité à travers les exonérations, des enclaves étrangères, de l'acculturation et de l'environnement.

En effet, les retombées économiques du tourisme balnéaire profitent peu aux populations locales. Il est d'ailleurs en train de vivre la plus grave crise de son histoire en raison des mécanismes de domination économique et de dépendance sur lesquels l'activité s'est développée. Tous les investissements proviennent de l'extérieur. Les clientèles sont également en majorité envoyées par les voyagistes français, italiens ou espagnols qui ont mis en place un circuit économique fermé qu'ils contrôlent du début à la fin. Pour mieux les contenir dans leur bulle, les voyagistes préparent les touristes à éviter les éventuels contacts avec le milieu extérieur au site d'hébergement et de villégiature. À l'intérieur des hôtels de type "tout inclus», les loisirs sont organisés de manière à ce que les touristes ne ressentent nullement la nécessité de sortir de leur "paradis».

Une grosse partie des bénéfices générés par l'activité appartient à ces multinationales étrangères qui contrôlent la quasi-totalité des structures hôtelières littorales où sont favorisés en vase clos des modes de vie des Français, Italiens ou Espagnols fondés sur leur propre système de références et sur leur interprétation du désir des touristes. Dans l'ensemble, le contrôle de l'activité touristique échappe aux pouvoirs publics locaux dont le rôle ne se limite qu'à la perception de taxes et d'impôts.

Aujourd'hui, la situation globale du tourisme balnéaire est devenue alarmante. Les flux touristiques ne cessent de dégringoler, voire de stagner. Le tourisme sénégalais, notamment le balnéaire, est confronté à d'énormes difficultés, au point que les acteurs sont unanimes à penser que c'est tout «le secteur qui est malade» (N'diaye Kopa, 2012 : communications personnelles). Depuis quelques années, l'État et les professionnels cherchent éperdument à soigner l'image du pays à l'extérieur, mais également à mettre en place une politique cohérente, 
dynamique, pour relever le secteur de sa léthargie totale. Des plans et programmes semblent être pilotés à vue sans planification stratégique réelle de l'activité.

Par ailleurs, sur le plan des investissements touristiques, le tableau 1 indique que les étrangers se taillent la part du lion, même si la plupart des campements et des auberges sont la propriété de nationaux en raison des faibles coûts d'investissements qu'ils nécessitent.

Les modalités de développement actuel de l'activité posent de profonds déséquilibres socio-économiques. En analysant la valeur et la richesse des potentialités touristiques et en les comparant avec les recettes générées par le tourisme balnéaire, on est frappé par le niveau de pauvreté des populations locales, tant en milieu urbain qu'en milieu rural. Dans les zones à vocation touristique, elles ne comprennent pas encore ce qu'est le tourisme.

En effet, les industries nationales sont exclues de la compétition au profit de grandes firmes internationales. Les circuits d'approvisionnement, très rétrécis, adaptés à une population réduite, sont délaissés en faveur des importations. À ce sujet, l'exemple du Club Aldiana situé près du village traditionnel de Nianing représente une parfaite illustration de ce phénomène paradoxal. Cette structure d'accueil d'une capacité de 950 clients importait la quasi-totalité de ses produits de consommation au moment où pourrissaient, juste à côté, dans le village, les produits de la ferme agricole entretenue par Caritas à cause du manque de débouchés ou de clients.

Il en est de même pour la pêche artisanale qui s'est détériorée parce que les pêcheurs, comme les paysans, ont été attirés soit vers les activités du bâtiment, soit vers des activités tertiaires comme la promenade en barque des touristes, l'exploitation de bars ou de restaurants, voire la création de campements, quand ils possèdent un lopin bien situé. Ils ne retournent au terroir qu'une fois la saison touristique terminée. Partout dans les villages littoraux, la vie rurale traditionnelle se modifie. Les anciens espaces agricoles ne sont plus en mesure de résister à l'extension rapide de l'espace touristique comme autour de Saly (Dehoorne et Diagne, 2008). La privatisation des plages, avec le prolongement des unités hôtelières et para-hôtelières sur la mer qui représente la principale zone de prédilection, entraîne une raréfaction des zones de débarquement. Par conséquent, la grande majorité des pêcheurs tournent le dos à l'océan, entraînant du coup la baisse des niveaux de production à Saly et à Ngaparou, par exemple. De plus, l'interdiction

\begin{tabular}{c|c|c}
\hline \multicolumn{3}{c}{ Tableau 1: Classement des réceptifs } \\
entre propriétaires nationaux et étrangers \\
\hline Types d'établissement & Nationaux & Étrangers \\
\hline Hôtels & 7 & 18 \\
\hline Résidences & 0 & 57 \\
\hline Campements et auberges & 49 & 12 \\
\hline Source : MTTA (2007 : 3). &
\end{tabular}

faite aux femmes de pratiquer des activités de séchage ou de fumage de poissons, considérées comme des nuisances pour les touristes dans les zones consacrées à l'activité touristique, ne fait qu'accentuer les inégalités quant à l'occupation de cet espace (Faye, $2005: 18$ ).

Les besoins des établissements touristiques ont été si massifs que s'est développée une forte immigration de travailleurs. Pourtant, tourisme et agriculture peuvent trouver une parfaite symbiose en ce sens que les métiers du tourisme et les travaux agricoles se succèdent dans le calendrier annuel (haute saison d'octobre à avril et basse saison de mai à novembre, ce qui correspond à la saison agricole). Toutefois, invité pour servir de panacée à la création d'emplois, le tourisme littoral n'est plus en mesure de répondre véritablement aux attentes des populations.

Les pertes d'emplois touristiques se chiffrent entre 2001 et 2007 à plus de $40 \%$ (N'diaye Kopa, 2012 : communications personnelles). En plus, la ségrégation sur le plan de l'emploi s'accentue entre expatriés et résidents locaux. Ces derniers, souvent employés par les hôtels comme des «éternels» saisonniers, sont réduits à n'obtenir que les emplois subalternes moins qualifiés et moins rémunérés. Les emplois qualifiés sont tenus par un personnel étranger, français notamment, car ils exigent un niveau de formation plus élevé que celui de la population locale (Renucci, $1990: 44$ ).

En effet, le développement du tourisme a très peu d'impact sur l'amélioration des conditions de vie des Sénégalais (Faye, 2005 : 18). Ses effets sur l'économie nationale restent donc faibles et ses performances sont très en deçà des potentialités du marché mondial du fait d'énormes difficultés et de contraintes à la fois structurelles, institutionnelles et conjoncturelles. Les indicateurs de performance sont également loin d'être satisfaisants avec, par exemple, des taux d'occupation de lits de $35 \%$ en 2010 et une durée de séjour qui tourne autour de 3 à 4 jours (MAT, $2010: 3$ ).

Le tourisme de masse a profondément contribué au bouleversement de la société sénégalaise, et littorale en particulier. Son développement a provoqué une mise en tourisme des cultures locales qui participe à leur «folklorisation» (Rozenberg, $1981: 9$ ). Il a éliminé les vielles habitudes traditionnelles en modernisant l'habitat et en facilitant la pénétration des modes de vie des sociétés occidentales. Les contacts entre touristes et habitants locaux étaient jadis très faciles, car ils étaient attachés aux vieilles valeurs d'hospitalité. Cependant, aujourd'hui, avec la prolifération effrénée des installations hôtelières et d'autres infrastructures touristiques au seul bénéfice des touristes, des manifestations d'hostilité se multiplient de la part de divers groupes de pression comme des associations de jeunes et de femmes protestant contre la mainmise d'étrangers sur leur terre. En même temps, il n'est pas rare de voir certaines personnes s'insurger contre des touristes pour la simple visite d'un lieu public ou la participation à une fête locale.

Par ailleurs, la hausse du niveau de vie dans les zones touristiques littorales, avec les progrès de l'aisance et du bien-être induit par l'importance de l'argent, incite à la remise en cause du système de valeurs traditionnelles. La richesse était jadis fondée sur la terre et sur sa valeur agricole (Renucci, 1990 : 
28). Aujourd'hui, la valeur touristique des zones littorales procurent des gains énormes à ceux qui se lancent dans la spéculation foncière et immobilière (Rozenberg, 1981 : 11).

Le tourisme balnéaire a stimulé l'inflation dans les stations. Les prix s'envolent en même temps que la clientèle étrangère augmente. Or, il faut le reconnaître, l'augmentation des coûts peut chasser le touriste, attiré dans le passé par les tarifs modérés et la monnaie faible (Renucci, 1990 : 41).

Le tourisme a aussi contribué à transformer le caractère des régions littorales et à détruire le patrimoine culturel et environnemental. Il participe activement à l'urbanisation littorale. En effet, l'invasion touristique massive résultant d'un déferlement international où prédominent les clientèles européennes exerce une forte pression sur l'organisation de l'espace à cause de la poussée des équipements, créateurs de surcharges littorales. De plus, par diffusion progressive à partir des noyaux touristiques que représentent les stations de Saly et du Cap Skirring, se développent de nouvelles «banlieues touristiques» sur les rivages qui intensifient l'occupation littorale, un problème déjà observé par Paskoff (1998: 16) et qui se poursuit aujourd'hui. L'attraction de base qui s'articule autour du rivage et de la vue sur la mer entraîne également une «course» à son occupation et au panorama. Le plus souvent, les touristes résidents qui s'établissent à Saly sont des retraités aisés et des couples à la recherche de l'exotisme. Ils sont séduits par le calme, la beauté du cadre et la douceur du climat. Toutefois, avec la flambée des prix des villas et des appartements meublés, ils conquièrent les zones périphériques des villages traditionnels littoraux, voire les cordons littoraux, les lagunes et les lacs qui sont à la fois étendus et peu denses.

La concentration spatiale des touristes sur le seul littoral accentue non seulement les processus de dégradation des ressources naturelles et environnementales mais aussi l'urbanisation. Il suffit d'observer la densité moyenne dans les villes touristiques littorales pour voir l'importance de l'urbanisation qui touche $56 \%$ du rivage (GIRMAC, 2004 : 9). Les centres urbains littoraux concentrent déjà $75 \%$ de la population et $85 \%$ de l'activité économique (GIRMAC, 2004 : 9). Cette dynamique urbaine a atteint un tel niveau à partir de 1990 que la saturation littorale des stations est devenue un véritable problème. À Saly, par exemple, la population saisonnière atteindrait 65000 habitants, alors que celle résidente n'est estimée qu'à 25000 habitants, ce qui représente presque le triple (N'diaye Kopa, 2012 : communications personnelles).

La «baléarisation» (Duhamel et Knafou, 2003 : 48) s'accentue sur cette portion de côte provoquant une ségrégation socio-spatiale qui s'accroît. La croissance des surfaces bâties s'effectue sans réel respect des normes et politiques, tant en matière d'urbanisation que des articulations entre la fonction résidentielle et la fonction de production. Ainsi se crée une nouvelle stratification socio-spatiale opposant bas quartiers et beaux quartiers, entraînant une tyrannie du site et des coûts fonciers. La ségrégation spatiale se précise et se renforce, car le paramètre de base est la vue sur mer ou non. Les plages sont réservées aux riches et l'arrière-pays, aux "pauvres", souvent des locaux. Les rapports sociaux font apparaître des conflits récurrents, d'ordre culturel, entre semi-résidents et population locale.
Les acteurs touristiques constatent une baisse progressive de l'activité touristique au fur et à mesure que la zone littorale se convertit en quartier résidentiel, lui faisant perdre sa première vocation (Équipe MIT : 2002 : 31; Duhamel et Knafou, 2003 : 47). Plusieurs semi-résidents louent des appartements, des villas ou des studios meublés à des Européens par Internet durant leur absence. Le recul du tourisme international se note à travers le fléchissement des fréquentations touristiques dans les établissements hôteliers. Le raz-de-marée immobilier, témoin d'un «modèle spéculatif lourd» encouragé par l'État, a fini par s'imposer dans toutes les stations littorales, faute de réglementation quant à la mise en location touristique. Il participe fortement à l'encombrement croissant et à la dégradation subséquente de l'environnement littoral (Duhamel et Knafou, 2003 : 51).

Inquiets du recul de l'activité touristique, les hôteliers accusent l'État d'être le seul responsable de cette situation en autorisant les résidences para-hôtelières sur le littoral. Les autorités s'activent à chercher des voies de solution, notamment par la diversification du produit. À ce propos, diverses actions de promotion sont menées à l'étranger. Cependant, ces mesures seront-elles suffisantes alors que le pays doit faire face à une concurrence vive? L'atteinte de l'objectif de 1500000 touristes (MTTA, 2005 :18) passe nécessairement par une meilleure planification et une intégration du secteur dans une dynamique de développement durable.

Le tourisme est généralement considéré comme une activité de sociabilité et de rencontre qui ne saurait se développer sans les populations d'accueil. En effet, il est généralement admis que lorsque les populations locales sont responsabilisées, elles s'investissent mieux dans les projets de développement qui touchent directement leur vécu.

Toutefois, le constat fait jusqu'ici révèle que les possibilités actuelles d'implication des communautés locales dans le processus de développement touristique sont limitées. Ces sites balnéaires à travers les structures internationales s'inscrivent dans une logique internationale où les perspectives de participation des populations sont réduites. C'est dans ce contexte que s'impose la réflexion sur la recherche de nouvelles alternatives mieux ancrées dans les territoires et les sociétés d'accueil pour un véritable projet de développement touristique durable. Dès lors, l'écotourisme est au cœur des nouvelles propositions (Dehoorne et al., 2007) en permettant de poser les jalons d'un tourisme profitable aux communautés locales.

\section{La mise en place d'un tourisme alternatif durable : l'exemple de l'écotourisme}

Le tourisme est certes une source de gains, mais il impose de lourdes dépenses aux pays et aux régions concernées, tant en termes de promotion que d'entretien des infrastructures qui lui sont destinées. Le tourisme au Sénégal crée un effet dualiste et antinomique. Les succès et les problèmes résultant de la mise en tourisme se résument tant en termes de retombées positives qu'en termes de perturbations socioéconomiques et environnementales.

Le tourisme littoral a contribué à donner conscience de l'identité et des responsabilités aux populations des zones dans lesquelles il s'est développé. C’est ainsi que dans le Delta du 


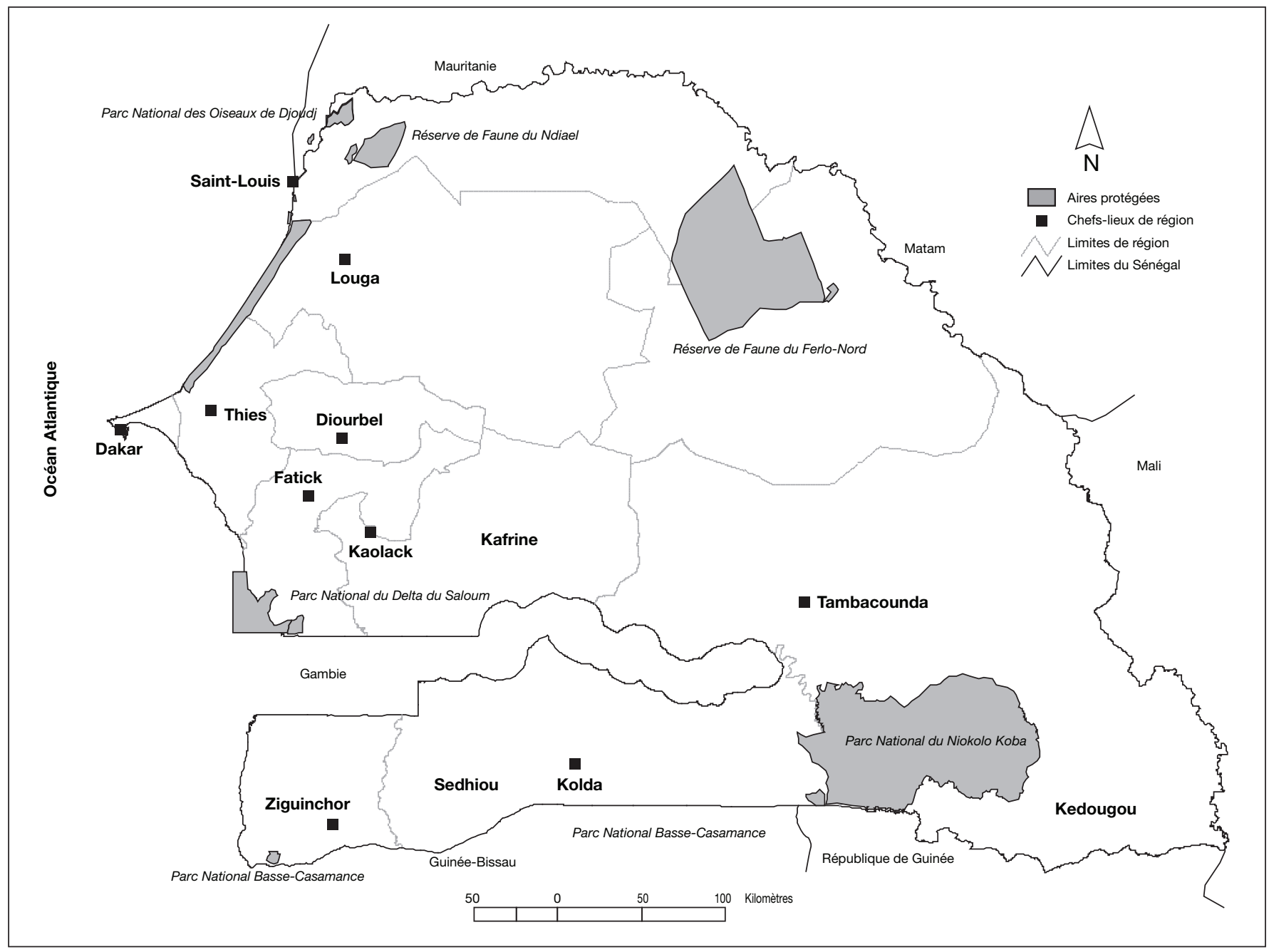

ILLUSTRATION 4 : Quelques potentialités écotouristiques du Sénégal (source : compilation de l'auteur à partir des données de DTGC (2010)).

Saloum, par exemple, il a aidé à l'affirmation d'une conscience régionale et de la nécessité de gérer l'espace, le capital nature. Dans ce contexte, le changement de l'offre touristique principale est-il possible?

Le Sénégal offre une gamme de produits touristiques liée à la richesse et à la diversité de ses milieux naturels et culturels. Ces potentialités naturelles constituent une niche écologique contribuant à maintenir la biodiversité avec des composantes en interrelation qui fondent son microclimat (les trois systèmes dunaires blanches, jaunes et rouges), la végétation, les eaux douces, et les eaux salées. La Langue de Barbarie, la lagune de Somone et Mbodienne, les sites sacrés de la PetiteCôte, du Sine Saloum et de la Casamance, les sites archéologiques de Diorom Boumack, les villages traditionnels du Delta, de la Petite-Côte, de la Casamance et du pays Bassari, et le littoral offrent des possibilités de découverte, de balade, et de randonnées équestres ou pédestres ou d'activités aquatiques (voir illustration 4).

La fréquentation touristique littorale peut être limitée, à travers la définition de la capacité de charge de chaque site, pour éviter un certain nombre de conflits récurrents liés à l'usage du sol. Il est tout aussi nécessaire de rechercher, au-delà des transformations de la société locale littorale sur près d'un quart de siècle de tourisme intensif, des variantes susceptibles de structurer durablement l'activité touristique au Sénégal.

Dès lors, l'intérêt majeur est porté sur l'écotourisme. Le ministère du Tourisme a pris conscience que le développement du secteur est une affaire de mieux-être pour les populations et que ce mieux-être passe par la préservation des ressources globales, l'évaluation des risques et la recherche d'une meilleure utilisation de l'espace (Beteille, $1996: 16$ ).

En d'autres termes, l'écotourisme permet d'éviter la dégradation des milieux fragiles. Il couvre les randonnées et les observations de la nature. Ces formes de loisirs attestent d'un souci de respecter l'environnement. Il s'agit notamment d'exploiter de façon optimale les ressources de l'environnement qui constituent un élément clé de mise en valeur touristique, en préservant les processus écologiques essentiels et en aidant à sauvegarder les ressources naturelles et la 
biodiversité. Cette recherche d'équilibre favorise un type de tourisme durable où les modes de consommation tiennent compte des possibilités à long terme.

Il est évident que la marche reste longue, mais le tourisme sénégalais peut saisir les opportunités qu'ouvre l'écotourisme grâce à sa tradition de protection de la nature. Les activités de tourisme en milieux naturels peuvent couvrir au total 1613790 ha, soit environ $8 \%$ du territoire national. D'ailleurs, en Afrique de l'Ouest, le Sénégal reste l'un des rares pays qui peut offrir en dehors du balnéaire autant de produits touristiques écologiquement et culturellement riches. L'atteinte des objectifs d'un tourisme maîtrisé à travers les flux de visiteurs, sain, respectueux des mœurs et profitable à l'économie, passe nécessairement par un tourisme plus soutenable et l'émergence d'activités touristiques novatrices capables de retenir les populations dans leur terroir (Duval, 2008:8).

Dans ces conditions, l'application d'une nouvelle politique d'aménagement et de gestion touristique s'impose pour éviter l'étouffement de la zone littorale, la stagnation ou le déclin de l'activité. L'utilisation d'une étude d'impact sur l'environnement devient nécessaire avant l'implantation d'un réceptif hôtelier. L'absence de telles études est préjudiciable au littoral qui est menacé par les investissements. Le respect des normes d'implantation sécurise l'insertion du tissu hôtelier.

Aussi, les ressources naturelles sont pour l'essentiel les matières premières du tourisme sénégalais (faune, flore, plage et climat). Il faut donc songer à les préserver. Il est indispensable d'envisager de nouvelles approches touristiques, complémentaires et originales, mieux intégrées aux milieux et aux sociétés d'accueil (Dehoorne et al., 2007). À cet égard, il s'avère impératif de s'inscrire dans une logique durable. L'accroissement du nombre de touristes, plus de 20000 par année au cours des années 1980 et 1990, consécutif au développement et à la qualité des infrastructures d'accueil a provoqué une certaine pression sur les ressources naturelles, notamment l'eau, l'énergie et la faune. Pour faire face à cette situation, le Sénégal a opté pour une réorientation de sa politique touristique à travers la vulgarisation de l'écotourisme qui représente un remède durable pour allier développement économique et protection écologique.

D'emblée, parler de l'écotourisme, c'est évoquer un concept holistique, une activité globalisante. Le terme est susceptible de recevoir des acceptations diverses, puisque les activités écotouristiques sont de formes multiples.

$\mathrm{Au}$ regard de ces considérations, est-il possible de spécifier le concept à travers une notion valablement acceptée? Dans tous les cas, on retiendra ici que le tourisme écologique respecte la nature et assure un développement soutenable. Il contrôle, réduit les déchets, les produits nocifs, et matériaux toxiques, corrosifs, infectieux ou inflammables. Il gère les réserves d'eau douce et le déversement des eaux résiduaires. Il est sensible à la conservation des espaces protégés ou menacés, à l'esthétique du paysage, améliore le milieu naturel et s'intéresse aux aspects environnementaux. À cet effet, on peut dire que la réussite touristique nécessite désormais une vocation solide, des compétences professionnelles avérées, tant pour l'aménagement, la production que la commercialisation, car les enjeux économiques et environnementaux se situent à tous ces niveaux-là. C'est pourquoi une éducation à l'environnement est une exigence. Comme le déclare LozatoGiotart (2006:7) : «Sur le chemin de l'écotourisme, seule une étude approfondie des enjeux environnementaux et de la prise en compte des notions de qualité et de durabilité touristique permettra d'imaginer un futur touristique.»

À ce propos, le tourisme étant une ressource économique de premier plan pour le Sénégal, il mérite d'être soutenu, ce qui ne se fera pas sans une prise en compte de l'environnement. Pour ce faire, il s'avère nécessaire de savoir comment on peut tirer un meilleur parti de cette activité pour les sociétés locales et mieux gérer les ressources environnementales dans une perspective durable.

Face à l'érosion de l'image des stations littorales sénégalaises, plus particulièrement Saly et Cap Skirring, les officiels du tourisme ont suscité, avec de très faibles moyens, des initiatives répondant à rééquilibrer l'activité sur le territoire national et à diversifier le produit touristique. Malgré la prééminence du littoral, des stratégies de développement touristiques préconisent une meilleure répartition sur le territoire des équipements touristiques.

Le gouvernement sénégalais pourrait ainsi inviter l'ensemble des acteurs touristiques à réfléchir sur les orientations à privilégier pour asseoir le tourisme durable au niveau de la «destination Sénégal».

Sur le plan international, l'écotourisme et le tourisme culturel ont enregistré une croissance rapide durant la dernière décennie et comptent pour $7 \%$ des échanges mondiaux, fournissant 195 milliards de dollars (OMT et PNUE, 2002 : 113). Pour le Sénégal, les opportunités à saisir dans le développement de cette forme de tourisme sont énormes, eu égard aux immenses potentialités écologiques et culturelles dont il regorge.

Par ailleurs, dans le cadre de la Stratégie de croissance accélérée, le tourisme figure parmi les secteurs porteurs de croissance et créateurs de richesses à travers la grappe «Tourisme, industries culturelles, artisanat d'art».

Dans ce contexte, le Sénégal réinvente une nouvelle politique touristique qui tend vers la diversification des produits proposés. Cette orientation permet d'atteindre quatre objectifs principaux. Il s'agit d'abord de préserver l'immense potentiel historique, écologique, ethnique et culturel du pays, ensuite de valoriser et d'exploiter judicieusement ces atouts, puis de mieux vendre la destination Sénégal à travers le monde, et enfin de faire bénéficier aux populations locales des retombées du tourisme.

Le tourisme durable valorise les atouts culturels et traditionnels et contribue à l'entente et à la tolérance interculturelle. Il ne connaît ni choc de cultures ni phénomène de rejet réciproque de réalités culturelles même dissemblables. Il s'épanouit plutôt dans le dialogue des cultures. Au même titre que l'environnement, le potentiel socioculturel constitue un substrat ou fondement de l'activité touristique durable. Il s'agit d'envisager une activité économique viable sur le long terme, offrant à toutes les parties prenantes des avantages socio-économiques réels et équitablement répartis en termes de création d'emplois stables, de possibilités de bénéfices et de services sociaux pour les communautés d'accueil, en somme 
une contribution à la réduction de la pauvreté. La préservation des ressources naturelles et culturelles permet de gérer durablement dans la mesure où elle est un atout immense dans le développement du secteur.

\section{Conclusion}

Le tourisme sénégalais est en quête d'une nouvelle identité pour plusieurs raisons que nous venons d'évoquer. En gros, il y a une forte concentration de l'activité touristique sur le littoral, ce qui est à l'origine de l'occupation anarchique de l'espace qui n'a profité qu'aux promoteurs immobiliers et aux multinationales étrangères. C'est l'une des raisons qui justifient cet essoufflement du tourisme balnéaire.

Dès lors, le contexte ne saurait être mieux choisi pour le littoral, bastion du tourisme de loisirs, pour opérer une mutation parfaitement en phase avec les enjeux mondiaux actuels et futurs. Cette nouvelle orientation, du reste inévitable, trouve également son explication au niveau local. Quarante années de tourisme balnéaire n'ont pas manqué d'occasionner des dommages au triple plan environnemental, socioculturel et économique sur toute l'étendue du littoral sénégalais, au point même que certains en arrivent à établir une relation de cause à effet entre tourisme et pauvreté dans ces zones côtières très vulnérables. Une certaine forme de tourisme et pauvreté, devrait-on dire, d'autant plus que l'activité touristique ne peut être uniquement réduite au balnéaire. Bien au contraire, car d'autres formes de tourisme, moins agressives mais plus durables, existent, parce que très soucieuses du respect de l'environnement et des retombées générées au bénéfice des populations.

Alors, plutôt que d'être un moyen de concurrencer le balnéaire, l'écotourisme reste et demeure la matrice du tourisme sénégalais, un complément indispensable qui est apte à prolonger le séjour des touristes, mais surtout à perpétuer l'activité touristique même.

\section{Références}

AMIROU, Rachid (1995) Imaginaire touristique et sociabilité du voyage,

Paris : Presses Universitaires de France (PUF). 281 p.

BETEILLE, Roger (1996) Tourisme vert, Paris : Presses Universitaires de France (PUF), Coll. «Que sais-je?». 127 p.

CORBIN, Alain (1988) Le territoire du vide. L'Occident et le désir du rivage 1750-1840, Paris : Aubier, Coll. «Historique». 416 p.

DEHOORNE, Olivier et Abdou Khadre DIAGNE (2008) «Tourisme, développement et enjeux politiques : l'exemple de la Petite Côte (Sénégal) ", Études caribéennes, 9-10/2008, Le tourisme dans les îles et littoraux tropicaux et subtropicaux, mis en ligne le 8 septembre 2008.

$<\mathrm{http}$ ://etudescaribeennes.revues.org/document1172.html $>$, consulté le 25 novembre 2008.

DEHOORNE, Olivier; Pascal SAFFACHE et Dominique AUGIER (2007)

"Tourisme, écotourisme et stratégie de développement dans la Caraïbe», Études caribéennes, $\mathrm{n}^{\circ} 6,<\mathrm{http} / / /$ etudescaribeennes.revues.org/3383>, consulté le 10 janvier 2012.

DÈME, Mouhamed Faouzou (2002) «Tourisme : ce qu'il en coûte aux Sénégalais », le quotidien Wal Fadjri, du 8 au 9 mai, p. 11-12.

DEWAILLY, Jean-Michel (2005) «Mise en tourisme et touristification», DANS AMIROU, Rachid; Philippe BACHIMON; JeanMichel DEWAILLY et Jacques MALÉZIEUX (sous la direction de),
Tourisme et souci de l'autre. En hommage à Georges Cazes, p. 1-18. Paris : L'Harmattan, Coll. «Tourismes et sociétés». 362 p.

DIOMBÉRA, Mamadou (2010) «Aménagement et gestion touristique durable du littoral sénégalais de la Petite Côte et de la Basse Casamance». Thèse de doctorat en tourisme, Saint-Louis (Sénégal) : Université Gaston Berger. 343 p.

DTGC — Direction des Travaux Géographiques et Cartographiques (2010) «Subdivision administrative du Sénégal», ANAT Gouvernement du Sénégal, Dakar, <http://dtgc.au-senegal.com/base.html>, consulté le 8 décembre 2012.

DUHAMEL, Philippe et Remy KNAFOU (2003) «Tourisme et littoral : intérêts et limites d'une mise en relation ", Annales de Géographie, vol. 112, $\mathrm{n}^{\circ} 629$, p. 47-67.

DUMAS, Danielle (1980) «Le Tourisme international dans le Bassin Méditerranéen. Situation actuelle et évolution récente», Information Géographique, n 5, p. 191-198.

DUVAL, Gérard (2008) «Demain, la fracture touristique?», Revue Espace, $\mathrm{n}^{\circ} 258$, p. 8-12.

Équipe MIT (2002) Tourismes Lieux communs, Paris : Belin, Coll. «Mappemonde». 320 p.

FAYE, Paul (2005) «Paupérisation des populations locales par le tourisme international de masse : Quelle interaction entre le DSRP et les politiques touristiques au Sénégal», Le Journal, lundi 13 juin, p. 18-19.

GIRMAC (2004) «Gestion intégrée du Littoral sénégalais», Rapport ministère de l'Environnement et de la Protection de la Nature, Dakar. 74 p.

LOZATO-GIOTART, Jean-Pierre (2006) Le chemin vers l'écotourisme : impacts et enjeux environnementaux du tourisme aujourd'hui, Paris : Delachaux et Niestlé SA. 192 p.

MAT - Ministère de l'Artisanat et du Tourisme (2010) «Conseil interministériel sur la relance du tourisme sénégalais», Dakar : MAT. 26 p.

MEF - Ministère de l'Économie et des Finances (2003) «Situation économique du Sénégal», Dakar : MEF. 145 p.

MTTA — Ministère du Tourisme et des Transports Aériens (2005) «Lettre de Politique Sectorielle de Développement du Tourisme», Dakar : MTTA. $32 \mathrm{p}$.

MTTA — Ministère du Tourisme et des Transports Aériens (2007) «Bulletin des statistiques touristiques », Direction des Études et de la Planification, MTTA. 8 p.

N'DIAYE KOPA, Doudou (2012) Chef du Bureau Communal du Tourisme de Mbour, entrevue réalisée à Mbour (Saly), le 9 janvier.

OMT — Organisation mondiale du tourisme (2003) Étude d'impacts du Tourisme sur l'Économie sénégalaise, Madrid, Rapport final (SEN 01/002). $144 \mathrm{p}$.

OMT et PNUE - Organisation mondiale du tourisme et Programme des Nations unies pour l'environnement (2002) «Sommet Mondial de l'Écotourisme : rapport final», Madrid, Espagne : World Tourism Organization. $113 \mathrm{p}$.

PASKOFF, Roland (1998) Les littoraux, Impacts des aménagements sur leur évolution, Paris : Armand Colin. 260 p.

QUASHIE, Hélène (2009) «Désillusions et stigmates de l'érotisme : Quotidiens d'immersions culturelles et touristiques au Sénégal», Cahiers d'études africaines, $\mathrm{n}^{\circ}$ 193-194, p. 525-550.

RENUCCI, Janine (1990) «Tourisme international et tourisme national dans les États de L'Europe méridionale», Annales de Géographie, vol. 99, $\mathrm{n}^{\circ} 551$, p. $21-50$.

ROZENBERG, Danielle (1981) Tourisme de masse et culture locale aux Baléares. Problèmes Politiques et Sociaux, Paris : La Documentation française, $\mathrm{n}^{\circ} 423.40 \mathrm{p}$. 